\title{
t-motives: Hodge structures, transcendence and other motivic aspects
}

\author{
G. Böckle, D. Goss, U. Hartl, M. Papanikolas
}

December 6, 2009

\section{Overview of the field}

Drinfeld in 1974, in his seminal paper [10], revolutionized the contribution to arithmetic of the area of global function fields. He introduced a function field analog of elliptic curves over number fields. These analogs are now called Drinfeld modules. For him and for many subsequent developments in the theory of automorphic forms over function fields, their main use was in the exploration of the global Langlands conjecture over function fields. One of its predictions is a correspondence between automorphic forms and Galois representations. The deep insight of Drinfeld was that the moduli spaces of Drinfeld modules can be assembled in a certain tower such that the corresponding direct limit of the associated $\ell$-adic cohomologies would be an automorphic representation which at the same time carries a Galois action. This would allow him to realize the correspondence conjectured by Langlands in geometry. Building on this, in [11] Drinfeld himself proved the global Langlands' correspondence for function fields for $\mathrm{GL}_{2}$ and later in [18] Lafforgue obtained the result for all $\mathrm{GL}_{n}$.

In a second direction, the analogy of Drinfeld modules with elliptic curves over number fields made them interesting objects to be studied on their own right. One could study torsion points and Galois representations, one could define cohomology theories such as de Rham or Betti cohomology and thus investigate their periods as well as transcendence questions. A main advance in this direction is the introduction of $t$-motives by Anderson in [1]. Passing from Drinfeld modules to $t$-motives may be compared to the passage from elliptic curves to abelian varieties. But more is true. The category of $t$-motives is also a simple function field analog of Grothendieck's conjectured category of motives over number fields. It is this second direction which constitutes the main theme of the present workshop on $t$-motives. Intended subtopics were Galois representations, $L$-functions, transcendence results, Hodge structures and period domains.

\section{Recent progress and open questions}

The past decade has seen many exciting new developments in the arithmetic of functions fields centered around the notion of a $t$-motive. Some of the most important ones are:

(1) New developments in the transcendence theory over function fields: For instance, it has been shown that the period matrix of a $t$-motive has transcendence degree equal to the dimension of its motivic Galois group, much in the same way that Grothendieck's conjecture predicts the transcendence degree of the period matrix of an abelian variety to be equal to the dimension of its Mumford-Tate group. 
(2) Hodge structures for function fields: Defined by Pink in 1997, they allow him to define the analog of the Mumford-Tate group of a $t$-motive and to formulate a Mumford-Tate conjecture for certain $t$-motives and to prove the conjecture for Drinfeld modules. Also Pink proved the Hodge-conjecture in this theory, stating, that the Mumford-Tate group is equal to the motivc Galois group.

(3) Period domains: More recently Pink's Hodge structures have been used extensively to lay foundations for period domains over function fields and state an analog of Fontaine's theory of crystalline Galois representations.

(4) Galois representations: While the Tate-conjecture for $t$-motives has been proved already in the early 90 's, only recently results on the openness of the image ( $l$-adically and adelically) have been obtained.

(5) Tannakian formalisms: Such have recently been described for t-motives in various contexts. They should ultimately link transcendence, Hodge structures and images of Galois representations.

(6) $L$-series: There are now cohomological approaches to $L$-series attached to $t$-motives. Moreover, recently new results on the zeroes of these $L$-series have emerged.

The above topics are tightly interwoven. The Tannakian formalism is used in transcendence theory as well as in a formulation of a Mumford-Tate conjecture based on function field Hodge structures (which is proved for Drinfeld modules). This in turn spurs the interest in Galois representations over function fields. All of the above topics have close relations to similar questions in number theory. The function field Hodge structures and analogs of Fontaine's theory have influenced questions on period spaces for number fields. Other developments such as the transcendence theory have gone far beyond comparable results in number theory. Let us focus on some particular topics:

\section{Transcendence}

New developments in the transcendence theory over function fields have led to important results on algebraic independence that as yet are out of reach over number fields. In the 1980's and 1990's work of Brownawell, David, Denis, Thakur, Yu, and many others demonstrated that there is a rich transcendence theory of exponentials and logarithms of Drinfeld modules that parallels ideas and results over number fields. This culminated with Yu's Sub-t-module Theorem [26], which characterized completely linear relations among logarithms of algebraic points on Drinfeld modules and $t$-modules.

More recently, there have been important new discoveries toward algebraic independence. Work of Anderson, Brownawell, and Papanikolas [3] demonstrated that results on linear relations could be adapted to approach problems of algebraic independence, and they proved theorems on the algebraic independence of special values of the geometric function field $\Gamma$-function. In [20], Papanikolas showed that the period matrix of a $t$-motive has transcendence degree equal to the dimension of its motivic Galois group, much in the same way that Grothendieck's conjecture predicts the transcendence degree of the period matrix of an abelian variety to be equal to the dimension of its Mumford-Tate group. Using this point of view, Papanikolas proved the algebraic independence of Carlitz logarithms, and later Chang and $\mathrm{Yu}[9]$ have applied Papanikolas' results to prove the algebraic independence of Carlitz zeta values. Subsequent works by Chang, Papanikolas, Thakur, and Yu $[6,7,8]$ have utilized these techniques to determine all algebraic relations among various combinations of zeta values, $\Gamma$-values (arithmetic and geometric), and periods and logarithms of rank 2 Drinfeld modules without complex multiplication.

During the conference several speakers spoke about recent progress in transcendence. Chang, Thakur, Pellarin, and Yu each gave expository talks on different aspects of transcendence 
theory, including automata methods (Thakur), Mahler's method (Pellarin), and the sub-tmodule and difference equations methods (Chang, Yu). Bosser spoke about his joint work with Pellarin on Drinfeld quasi-modular forms [4, 5], which has been motivated by the still open problems concerning the algebraic independence of values of modular forms, and Chang spoke about some partial results in these directions. Chang also spoke on joint work with Papanikolas on recent results on algebraic independence of periods and logarithms of Drinfeld modules of arbitrary rank.

Although there has been much progress in function field transcendence during recent years, there are still many open problems. The challenge of determining all algebraic relations among periods and logarithms of arbitrary $t$-motives remains open. Adapting the current $\infty$-adic methods to problems in $v$-adic transcendence $(v$ a finite place) requires additional study. Proving a $t$-motivic version of Schanuel's conjecture for the classical exponential function also remains out of reach but yet poses many interesting projects.

\section{Hodge-Pink structures}

In [22] Pink has clarified the concept of Hodge structures in equal characteristic, which today are called Hodge-Pink structures. Using the theory of $\sigma$-bundles on the punctured open unit disc [17] he has defined the Hodge realization functor from uniformizable $t$-motives to HodgePink structures. This allows to associate to each uniformizable $t$-motive a Hodge group, also called Mumford-Tate group. In an unpublished work Pink proved that the Hodge group equals the motivic Galois group of the $t$-motive, yielding a precise analog of the famous Hodge conjecture, which is an outstanding open problem for varieties over the complex numbers. The above theory further enabled Pink to prove his celebrated analog of the Mumford-Tate Conjecture for Drinfeld modules [23]. It also allowed to transfer the notion of Fontaine's filtered isocrystals to the arithmetic of function fields, and the analogy of $p$-adic Hodge theory to emerge $([13,16])$. The latter is called Hodge-Pink theory. By a correspondence strongly paralleling the situation for abelian varieties, one can, in particular, associate to a $t$-motive with good reduction over a local field an isocrystal with Hodge-Pink structure. The latter structures possess period domains and the picture (see $[12,15]$ ) is much the same as the one in mixed characteristic developed by Rapoport, Zink, Faltings and others, which had great impact on the understanding of Shimura varieties and the Langlands program. Besides being a beautiful theory in its own right, the insights gained from Hodge-Pink theory have inspired recent work of Faltings and Hartl on classical $p$-adic Hodge theory, which has attracted much attention from researchers in number theory.

Despite this progress, many questions remain open. The Mumford-Tate conjecture for higher dimensional $t$-motives deserves further study, as well as its possible relations to transcendence theory. Semi-stable reduction of $t$-motives and their Hodge-Pink theory needs to be investigated. The cohomology of period domains and related spaces has not been considered much.

At the conference a lecture series by Hartl and Kim was devoted to explaning Pink's unpublished results on the Hodge realization functor and the Hodge conjecture, as well as the function field analog of Fontaine's theory of crystalline Galois representations.

\section{$L$-functions}

As in number theory, a fascinating topic are $L$-functions for function fields, and especially those which take values in positive characteristic [14]. By work of Taguchi and Wan [24] with methods in the spirit of Dwork, these $L$-functions are known to have an entire continuation to an analog of the complex plane in positive characteristic. A cohomological proof was later given by Böckle and Pink. But no functional equation is known. The special values of 
these $L$-functions at "even" positive integers are related to Bernoulli numbers over function fields. In the truely remarkable article [19], V. Lafforgue very recently proved an analog of the Bloch-Kato conjectures. For the $v$-adic (but so far not $\infty$-adic) $L$-series of Goss this does imply an interpretation of special values also at positive integers.

Lafforgue's result needs to be much further exploited. Perhaps it can help to shed some light on the rather elementary question of the number of zeros at the negative integers of $\zeta$-functions as raised in [25]. Until the workshop there was no analog of the Birch and Swinnerton-Dyer conjecture (BSD); despite the fact that $L$-series of Drinfeld modules and Drinfeld cusp forms are fairly well understood. In some cases the article [2] gave an interpretation of special values at 1 similar to the classical case. In this respect, the workshop really saw some new and very exciting development. In his talk at the workshop L. Taelman presented several ideas that very strongly point in the direction of a BSD-type conjecture.

\section{Impact and achievements of the workshop}

The workshop included most of the main researchers in the area. A main scholar missing was R. Pink from the ETH Zürich. Due to health problems he had cancelled his attendance only days before the workshop. This caused a serious problem for the planned lecture series on Hodge structures for functions fields which were discovered by Pink. We are grateful to U. Hartl who was able to substitute for Pink.

The workshop was attended by 35 participants. Among them were $7 \mathrm{PhD}$ students and 7 early postdocs. The workshop provided a marvelous occasion to bring together so many researchers in the area of function field arithmetic at one place to interact, to discuss and to make contacts. For the early postdocs and the $\mathrm{PhD}$ students this proved a unique opportunity to gain a broad and deep insight into many current aspects of the arithmetic of $t$-motives.

In this respect, we also believe that it was a very good idea to have a workshop program with a rather narrow focus, namely to concentrate on the arithmetic theory of $t$-motives and their various motivic aspects. This made it possible to present much of the state of the art in the area. But it also helped to have a focussed group of participants. All lectures had a large audience, the auditorium was always full.

The two lecture series which we had organized to expose some of the most active fields in the area and to disseminate the most recent theories and results turned out to be a great success. We thank the speakers in this lecture series Dale Brownawell, Chieh-Yu Chang, Urs Hartl, Wansu Kim, Federico Pellarin, Richard Pink (who planed to lecture but had to cancel due to illness), Dinesh Thakur, and Jing Yu for their excellent talks. The vast majority of the participants attended all talks in both lecture series: One on Hodge structures over function fields and one on transcendence in function field arithmetic. We believe that it was a great opportunity for both sides to learn from each other. The lecture series coming from different ends successfully build a bridge between the two topics. In one of the talks Hartl explained Pink's unpublished proof of the Hodge conjecture stating that the Mumford-Tate group by Pink agrees with the motivic Galois group defined by Papanikolas and Taelman. In another talk Chieh-Yu Chang explained how Pink's results on the monodromy of $\pi$-adic Galois representations associated to Drinfeld modules provided an important input for his joint results with M. Papanikolas on transcendence of periods of Drinfeld modules.

Outside the two lecture series there were many other interesting talks, most of an excellent quality. We have gathered titles and abstracts and summaries for talks without an abstract in Section 4. Here we only want to mention a few of the talks who perhaps stuck out most since their main results describe very recent developments that among many participants gained much attention: L. Taelman's talk gave a definition of a Mordell-Weil and a TateShafarevich group for $t$-motives and described some conjectures relating these to special 
values of the $L$-function of the $t$-motives. L. Taelman and D. Goss also started a project during the conference of generalizing some of the results to general $A$-motives. V. Bosser gave a talk on joint work with F. Pellarin on quasi-modular forms in the Drinfeldian setting. At the end he indicated a recent construction of Pellarin who defined a new kind of forms as deformations of classical Drinfeld quasi-modular forms. A. Pál announced some very strong results in the direction of Gross-Zagier for function fields. They improve results of Rück and Tipp and parallel unpublished research of Ulmer but the methods seem different. Wansu Kim used the analogs of Fontaine theory by Genestier-Lafforgue and Hartl to define and study universal deformation rings in the function field setting. Considering the success and importance such rings have for number fields, one can expect exciting developments.

In particular, the achievements of the present BIRS-workshop correspond well to the goals set in our proposal from two years back: (a) To present the state of the art of the arithmetic of $t$-motives in a single conference including related other recent topics in function field arithmetic. (b) To bring together the most active researchers to discuss further possible developments and the potential that lies in the recently developed methods. (c) To get a fair number of post-docs and recent $\mathrm{PhD}$ students in this and neighboring areas involved, among them in particular some that work in algebraic number theory. (d) To have two educational lecture series of 3-4 talks each, one on transcendence and another one Hodge structures and periods. (e) To enhance interaction among the participants on a personal level and in a stimulating environment in order to foster further joint projects.

We would like to end with a big thanks to the BIRS team for all their support regarding the organizational issues of the workshop. They provided much help and were always available. This made the stay of all the participants a really unique one. Many thanks to Brenda Williams, Danny Fan, and Wynne Fong and also to the director of BIRS, Dr. Nassif Ghoussoub.

\section{References}

[1] G. Anderson, t-Motives, Duke Math. J., 53 (1986), 457-502.

[2] G. W. Anderson, Log-algebraicity of twisted A-harmonic series and special values of L-series in characteristic p, J. Number Theory 60 (1996), no. 1, 165-209.

[3] G. W. Anderson, W. D. Brownawell, and M. A. Papanikolas, Determination of the algebraic relations among special $\Gamma$-values in positive characteristic, Ann. of Math. (2) 160 (2004), 237-313.

[4] V. Bosser and F. Pellarin, Hyperdifferential properties of Drinfeld quasi-modular forms, Int. Math. Res. Not. IMRN 2008, no. 11, art. ID rnn032, 56 pp.

[5] V. Bosser and F. Pellarin, On certain families of Drinfeld quasi-modular forms, J. Number Theory 129 (2009), 2952-2990.

[6] C. Y. Chang and M. A. Papanikolas, Algebraic relations among periods and logarithms of rank 2 Drinfeld modules, Amer. J. Math. (to appear).

[7] C. Y. Chang, M. A. Papanikolas, D. S. Thakur, and J. Yu, Algebraic independence of arithmetic Gamma values and Carlitz zeta values, Adv. Math. (to appear).

[8] C. Y. Chang, M. A. Papanikolas, and J. Yu, Geometric Gamma values and zeta values in positive characteristic, Int. Math. Res. Not. IMRN (to appear). 
[9] C. Y. Chang and J. Yu, Determination of algebraic relations among special zeta values in positive characteristic, Adv. Math. 216 (2007), 321-345.

[10] V. G. Drinfeld, Elliptic modules, Math. USSR Sbornik 23 (1974), 561 - 592.

[11] V. G. Drinfeld, Langlands' conjecture for GL(2) over functional fields, in "Proceedings of the International Congress of Mathematicians" (Helsinki, 1978), 565 - 574, Helsinki, 1980. Acad. Sci. Fennica.

[12] L. Fargues, A. Genestier, V. Lafforgue: L'isomorphisme entre les tours de Lubin-Tate et de Drinfield, Progress in Mathematics, Vol. 262, Birkhäuser Verlag, Basel 2007.

[13] A. Genestier, V. Lafforgue: Théorie de Fontaine en égales charactéristiques, Preprint 2008.

[14] D. Goss, L-series of t-motives and Drinfeld modules, in "The arithmetic of function fields (Columbus, OH, 1991)", 313-402, de Gruyter, Berlin, 1992.

[15] U. Görtz, T. Haines, R. Kottwitz, D. Reuman: Dimensions of some affine DeligneLusztig varieties, Ann. Sci. École Norm. Sup. (4) 39 (2006), no. 3, 467-511.

[16] U. Hartl: Period Spaces for Hodge Structures in Equal Characteristic, to appear in Ann. of Math. (2010), see also arXiv:math. NT/0511686.

[17] U. Hartl, R. Pink: Vector bundles with a Frobenius structure on the punctured unit disc, Comp. Math. 140 n.3 (2004), 689-716.

[18] L. Lafforgue, Chtoucas de Drinfeld et correspondance de Langlands, Invent. Math., 147 (2002), no. 1, 1-241.

[19] V. Lafforgue, Valeurs spéciales des fonctions L en caractéristique $p$, J. Number Theory 129 (2009), no. 10, 2600-2634.

[20] M. A. Papanikolas, Tannakian duality for Anderson-Drinfeld motives and algebraic independence of Carlitz logarithms, Inv. Math. 171 (2008), 123-174.

[21] F. Pellarin, Aspects de l'indépendance algébrique en caractéristique non nulle, Séminaire Bourbaki, Vol. 2006/2007, Astérisque No. 317 (2008), Exp. no. 973, vii, 205-242.

[22] R. Pink: Hodge Structures over Function Fields, Preprint 1997, available at http://www. math. ethz.ch/ pink.

[23] R. Pink: The Mumford-Tate conjecture for Drinfeld-modules, Publ. Res. Inst. Math. Sci. 33 (1997), no. 3, 393-425.

[24] Y. Taguchi, D. Wan, L-functions of $\varphi$-sheaves and Drinfeld modules, J. Amer. Math. Soc. 9 (1996), no. 3, 755-781.

[25] D. Thakur, On characteristic p zeta functions, Compositio Math. 99 (1995), no. 3, 231-247.

[26] J. Yu, Analytic homomorphisms into Drinfeld modules, Ann. of Math. (2) 145 (1997), 215-233. 


\section{Talks presented (in alphabetical order of speakers)}

Speaker: Vincent Bosser (Université de Caen)

Title: On Drinfeld quasi-modular forms

Abstract: In this talk, I will discuss some recent results involving Drinfeld quasi-modular forms that were obtained in a joint work with F. Pellarin. In particular, I will describe new advances in the problem of estimating the order of vanishing at infinity of such forms.

Speaker: Florian Breuer (University of Stellenbosch)

Title: The André-Oort Conjecture for Drinfeld modular varieties.

Abstract: We explore an analog of the André-Oort conjecture for subvarieties of Drinfeld modular varieties. The conjecture states that a subvariety $X$ of a Drinfeld modular variety contains a Zariski-dense set of complex multiplication $(\mathrm{CM})$ points if and only if $X$ is a "special" subvariety (i.e. $X$ is defined by requiring additional endomorphisms). We prove this conjecture in two cases. Firstly when $X$ contains a Zariski-dense set of CM points with a certain behaviour above a fixed prime (which is the case if these CM points lie in one Hecke orbit), and secondly when $X$ is a curve containing infinitely many CM points without any additional assumptions.

Speaker: Dale Brownawell (Penn State University)

Title: Introduction to Drinfeld modules and t-motives (Introductory lecture)

Summary of Talk: Brownawell gave the first talk of the conference, which was an introduction to Drinfeld modules, $t$-modules, and $t$-motives for non-specialists. He gave an overview of the different objects in the area, including exponential functions, periods, and $t$-motives themselves, and he compared the function field theory to the classical theories of commutative algebraic groups.

Speaker: Chieh-Yu Chang (NCTS, Taiwan)

Title: Frobenius difference equations and difference Galois groups (Transcendence lecture series)

Summary of Talk: Chang presented an expository lecture on the Galois theory of Frobenius semi-linear difference equations and their applications to transcendence problems for $t$-motives. He gave summaries of work of Anderson, Brownawell, and Papanikolas, and demonstrated their applicability to problems involving values of zeta functions and Gamma functions.

Speaker: Chieh-Yu Chang (NCTS, Taiwan)

Title: Algebraic relations among periods and logarithms for Drinfeld modules

Abstract: Given a Drinfeld $\mathbb{F}_{q}[t]$-module $\rho$ defined over an algebraic function field, we consider its period matrix $P$. We prove that all the algebraic relations among the entries of $P$ are those linear relations induced from the endomorphisms of $\rho$. We further prove the algebraic independence of $\rho$-logarithms of algebraic points that are linearly independent over the endomorphism ring of $\rho$. (This is a joint work with Matt Papanikolas). We will also present an application to the transcendence of certain special values of Drinfeld quasi-modular forms.

Speaker: Imin Chen (Simon Frazer University)

Title: Newton polygons of exponential functions attached to Drinfeld modules of rank 2

Abstract: Jointly with Yoonjin Lee we explicitly determine the Newton polygons of exponential functions attached to Drinfeld modules of rank 2 defined over $\mathbb{F}_{q}(T)$. The method is mostly elementary but nonetheless reveals some interesting closed form patterns. 
Speaker: Ernst-Ulrich Gekeler (Universität des Saarlandes)

Title: Frobenius actions on the cohomology of Drinfeld modules

Abstract: With (the motive of) a Drinfeld module one associates several modules or vector spaces that - in the familiar analogy of Drinfeld modules with abelian varieties - play the parts of singular, Betti, or deRham (co-)homology modules. We report on the relations between these, and study the various Frobenius actions in case the Drinfeld module is defined in finite characteristic.

Speaker: Urs Hartl (Universität Münster) substituting Richard Pink (ETH Zürich)

Title: Hodge structures over function fields I (Hodge structure lecture series)

Summary of Talk: Hartl presented Pink's theory in which he constructs a neutral Tannakian category of Hodge structures over the underlying function field. This allows in particular to define for each Hodge structure a Hodge group which is the function field analog of the Mumford-Tate group of a variety over the complex numbers. The talk further explained the Hodge realization functor which associates with each mixed uniformizable $t$-motive a mixed Hodge structure. For the construction of this functor the theory of $\sigma$-bundles on the punctured open unit disc is used.

Speaker: Urs Hartl (Universität Münster) substituting Richard Pink (ETH Zürich) Title: Hodge structures over function fields II (Hodge structure lecture series)

Summary of Talk: In this second talk on Pink's work Hartl explained the concept of $\sigma$-bundles on the punctured open unit disc and its classification theorem. He demonstrated how it is used to construct the Hodge realization functor. He also presented Pink's unpublished proof of the Hodge conjecture which states that the Hodge group of a $t$-motive equals the motivic Galois group as defined by Papanikolas and Taelman.

Speaker: Urs Hartl (Universität Münster)

Title: Divisible local t-motives, local shtukas and Hodge structures (Hodge structure lecture series)

Abstract: If one completes a $t$-motive at a finite place $v$ of the underlying function field one obtains what is called a local shtuka. Dually for an abelian $t$-module the $v^{n}$-torsion forms the divislble local $t$-motive, which is an analog of the $p$-divisible group associated with an abelian variety. Like $t$-motives and abelian $t$-modules, local shtukas and divisible local $t$-motives are antiequivalent concepts. More precisely, local shtukas are a kind of Dieudonné modules or displays for divisible local $t$-motives. Furthermore, in the arithmetic of function fields local shtukas play the role of Fontaine's crystalline Galois representations from $p$-adic Hodge theory. Via the analog of the mysterious functor of Grothendieck and Fontaine, local shtukas are classified by function field isocrystals with Hodge-Pink structure. Hodge-Pink structures were invented by Pink as a Hodge theory over function fields. To decide which isocrystals with Hodge-Pink structure arise from local shtukas there is a necessary numerical weak admissibility condition. Over omplete valued base fields with finitely generated value group this condition is also sufficient. This is the analog of the Colmez-Fontaine theorem stating that "weakly admissible implies admissible". Over algebraically closed base fields however, this is false.

Speaker: Toshiro Hiranouchi (RIMS, Kyoto University)

Title: Smallness of fundamental groups for varieties over finite fields

Abstract: It is known that the Hermite-Minkowski type theorem for curves over finite fields. It asserts that on a function field of one variable over a finite field, there exist only finitely many separable extensions of bounded degree and discriminant. In this talk, we will deal with higher dimensional analog of such theorem and its applications (joint work with S. Harada). 
Speaker: Wansu Kim (Imperial College, London)

Title: Weak admissibility, Galois Representations, and Deformations (Hodge structure lecture series)

Abstract: The first half of this lecture will be a continuation of Professor Hartl's lecture, focusing on the definition and foundational results on weak admissibility for isocrystals with Hodge-Pink structures and its relation with Galois representations, due to GenestierLafforgue, Hartl, etc.

In the second half of this lecture, I will discuss the existence and the local structure of the equicharacteristic analog of crystalline deformation rings, as discussed in my thesis. Mind that the existence of such equi-characteristic deformation rings may be surprising because a 'usual' deformation functor (with no deformation condition) has an infinite-dimensional tangent space in the equi-characteristic case, hence unlike the $p$-adic case we have no 'unrestricted' universal deformation ring to start with (among complete local noetherian rings). Thus, the key step in the proof of the existence is to show the finiteness of the tangent space of the deformation subfunctor defined a deformation condition analogous to torsion crystalline deformations with all weights within a fixed bounded interval.

The local structure of such equi-characteristic deformation rings is very similar to that of crystalline deformation rings studied by Kisin, and the proofs are also similar (except that in the equi-characteristic case we should work with weakly admissible isocrystals with HodgePink structures instead of weakly admissible filtered isocrystals).

If time allows, I would like to discuss some interesting open questions.

Speaker: Ignazio Longhi (National Taiwan University)

Title: On Iwasawa theory over function fields

Abstract: The goal is to present a short account of some recent developments in (abelian) Iwasawa theory for function fields in positive characteristic. In this setting, the role cyclotomic towers play over number fields is taken by the extensions arising from the torsion of Drinfeld-Hayes modules. The focus shall be on Iwasawa theoretic intepretations of $p$-adic $L$ functions for automorphic abelian varieties (with most emphasis on the elliptic curves case) and/or for Drinfeld-Hayes modules.

Speaker: Ambrus Pál (Imperial College London)

Title: Constructing rational points on genus one curves

Summary of Talk: Pál addressed the question whether a geometrically irreducible variety has a rational point over a solvable Galois extension of the ground field. He presented known results of others and new results of his own, as well as a strategy to approach this question using Drinfeld modular varieties. In this direction he announced strong results on the analogue of Gross-Zagier for function fields.

Speaker: Mihran Papikian (Penn State University)

Title: On the arithmetic of modular curves of D-elliptic sheaves

Abstract: The notion of $\mathcal{D}$-elliptic sheaf is a generalization of the notion of Drinfeld module. $\mathcal{D}$-elliptic sheaves and their moduli schemes were introduced by Laumon, Rapoport and Stuhler in their proof of certain cases of Langlands conjecture over function fields.

We discuss basic arithmetic properties of modular curves of $\mathcal{D}$-elliptic sheaves and draw parallels with the theory of Shimura curves. In particular, we produce a genus formula for modular curves of $\mathcal{D}$-elliptic sheaves, examine the existence of rational points on these curve, compute their fundamental domains in Bruhat-Tits trees, and determine the cases when these curves are hyperelliptic. As applications of previous results, we construct new asymptotically optimal sequences of curves over finite fields (such sequences are important in coding theory), 
and find presentations for certain arithmetic groups arising from quaternion algebras over function fields.

Speaker: Federico Pellarin (Universite Jean Monnet, St. Etienne)

Title: On Mahler's method (Transcendence lecture series)

Abstract: Mahler's method is a technique to show the transcendency of values of certain transcendental functions at algebraic complex numbers that was introduced by Mahler in 1929. These functions must satisfy functional equations in a certain class. Although transcendency of values of these functions can be also reached by other techniques (eg. Schmidt's subspace theorem), a remarkable feature of Mahler's method is that it also delivers algebraic independence in quite a precise way.

Moreover, the method extends to values of certain functions at points which are algebraic over the field $\mathbf{F}_{q}(\theta)$. Surprisingly, it turns out in this context that certain values of these functions are also "periods" of Anderson's $t$-modules. This was first remarked by Denis, and used to show algebraic independence of Carlitz logarithms of elements of $\mathbf{F}_{q}(\theta)$.

The aim of this lecture is to give a survey of this method, its interesting features, and its limits.

Speaker: Lenny Taelman (Mathematisch Instituut Leiden)

Title: The Mordell-Weil and Tate-Shafarevich modules of a Drinfeld module

Abstract: I will propose a definition of the Mordell-Weil and Tate-Shafarevich modules of a Drinfeld module with everywhere good reduction. These will be finitely generated, and finite, respectively. Moreover, I will explain how these can be used to give a class-number style formula for a certain special value of the Goss $L$-function of the Drinfeld module.

Speaker: Dinesh Thakur (University of Arizona)

Title: Automata methods in transcendence (Transcendence lecture series)

Summary of Talk: Thakur presented an expository lecture on automata methods in transcendence, which has applications to the transcendence of various quantities, including periods and values of Gamma functions. It has been known for sometime, by work of Christol et al., that algebraic power series are produced by finite automata. Thakur discussed how different types of automata and formal language theories provide a finer structure among transcendental numbers over function fields, which has led to many open problems.

Speaker: Dinesh Thakur (University of Arizona)

Title: Hypergeometric functions over function fields

Summary of Talk: Thakur presented recent work on function field hypergeometric functions, joint with Wen, Yao, and Zhao. He discussed two versions of hypergeometric functions, their connections with other analytic functions, such as exponentials of Drinfeld modules, and he gave criteria for the transcendence of their values.

Speaker: Seidai Yasuda (Kyoto University)

Title: Regulators, periods, and special vaules of automorphic $L$ functions over function fields. Abstract: In this talk, we give two formulas on special values on automorphic $L$ functions over $\mathrm{GL}_{n}$ over function fields. The first formula, obtained in a joint work with S. Kondo, involves a regulator map on a Drindeld modular variety whch establishes a function-field analog of Beilinson's conjecture. The second one involves an integral over the diagonal torus of a cusp form on $\mathrm{GL}_{n}$ over the rational function field.

Speaker: Jing Yu (National Taiwan University)

Title: On transcendence theory of Drinfeld modules (Transcendence lecture series) 
Summary of Talk: Yu presented an expository lecture on his Sub-t-module Theorem and its application to linear independence problems for periods and logarithms of $t$-modules. After giving an overview of the transcendence theory of Drinfeld modules and $t$-modules, which culminated in the Sub-t-module Theorem, he presented several applications to linear forms in logarithms and linear independence of Carlitz zeta values.

\section{$5 \quad$ List of Particpants}

Armana, Cécile, Universität des Saarlandes, Böckle, Gebhard, University of Duisburg-Essen, Bosser, Vincent, Université de Caen, Breuer, Florian, University of Stellenbosch, South Africa, Brownawell, Dale, Penn State University, Butenuth, Ralf, Universität Duisburg-Essen, Chang, Chieh-Yu, National Center for Theoretical Sciences Taiwan, Chen, Imin, Simon Fraser University, Cojocaru, Alina Carmen, University of Illionois at Chicago, Gekeler, Ernst-Ulrich, Universitt des Saarlandes, Goss, David, The Ohio State University, Hartl, Urs, University of Münster, Hiranouchi, Toshiro, RIMS, Kyoto university,

Hsia, Liang-Chung, National Central University Taiwan, Hubschmid, Patrik, ETH Zurich, Jeong, Sangtae, Inha University Korea, Karumbidza, Archiebold, University of Stellenbosch South Africa, Kim, Wansu, Imperial College London, Kondo, Satoshi, University of Tokyo, Laskar, Abhijit, Université de Strasbourg, Long Hoelscher, Jing, University of Arizona, Longhi, Ignazio, National Taiwan University, Lutes, Brad, Texas A \& $M$ University, Newton, James, Imperial College London, Pál, Ambrus, Imperial College London, Papanikolas, Matthew, Texas A $\& 3$ University, Papikian, Mihran, Pennsylvania State University, Pellarin, Federico, Universit Jean Monnet Saint-Etienne, Rück, Hans-Georg, Universität Kassel, Taelman, Lenny, Mathematisch Instituut Leiden NL, Thakur, Dinesh, University of Arizona, Top, Jaap, University of Groningen, Department of Mathematics, Wei, Fu-Tsun, National Tsing Hua University,

Yasuda, Seidai, Kyoto University, Yu, Jing, National Tsing-Hua University Taiwan, 